\title{
MATHEMATICAL ANALYSIS AND NUMERICAL METHODS FOR PRICING PENSION PLANS ALLOWING EARLY RETIREMENT*
}

\author{
M.C. CALVO-GARRIDO ${ }^{\dagger}$, A PASCUCCI ${ }^{\ddagger}$,AND C. VÁZQUEZ $\$$
}

\begin{abstract}
In this paper, we address the mathematical analysis and numerical solution of a model for pricing a defined benefit pension plan. More precisely, the benefits received by the member of the plan depend on the average salary and early retirement is allowed. Thus, the mathematical model is posed as an obstacle problem associated to a Kolmogorov equation in the time region where the salary is being averaged. Previously to the initial averaging date, a nonhomogeneous one factor Black-Scholes equation is posed. After stating the model, existence and regularity of solutions are studied. Moreover, appropriate numerical methods based on a Lagrange-Galerkin discretization and an augmented Lagrangian active set method are proposed. Finally, some numerical examples illustrate the performance of the numerical techniques and the properties of the solution and the free boundary.
\end{abstract}

Key words. retirement plans, options pricing, Kolmogorov equations, complementarity problem, numerical methods, augmented Lagrangian formulation

AMS subject classifications. 91G80, 35K65, 35K85, 65M25, 65M60, 90C33

1. Introduction. Pension plans are usually classified into defined contribution plans and defined benefit plans [5]. In the case of defined contribution ones, the employee receives at retirement an amount whose value depends on the investment earnings and the total contributions of both employer and employee to the employee pension plan account. Sometimes the employee can decide among the possible investments of the account, so that he/she bears the risk. In the defined benefit plans the pension at retirement depends on several factors: the number of years of service, the salary or the average salary, for example. A traditional defined benefit pension plan pays the salary at retirement multiplied by a number of years and by an accrued rate. Therefore, in this case the employer bears the liability, which is the amount of money that he/she has to set aside to fund the employee's retirement pension in the future. The value of this liability is what we refer here as the pension plan benefits or value. Although nowadays a large number of plans are accumulation plans, there is still a significant number of defined benefits plans as those ones here treated (as the Final Average Pay in USA, for example).

In this paper, we first pose a mathematical model to obtain the value of a defined benefit pension plan including early retirement option for the employee. More precisely, we assume that the amount received by the employee depends on the average salary corresponding to a certain number of years before retirement. In the case without the early retirement option opportunity, an analogous study has been addressed in [7]. In the present paper we incorporate the option of early retirement, which requires the application of similar modeling tools as those ones for pricing American options in quantitative finance. Therefore, a free boundary problem formulation naturally arises, the free boundary being an optimal retirement boundary between the region where it is optimal to retire and the region where it is optimal to continue working.

The departure point in the modeling is the consideration that the salary is stochastic, so that the pension plan can be handled as an option on the average salary. Then, the dynamic hedging methodology in

\footnotetext{
*This paper has been partially founded by MICINN (Project MTM2010-21135-C02-01) and by Xunta de Galicia (Project INCITE09105339PR and Ayuda CN2011/004 cofunded with FEDER funds).

${ }^{\dagger}$ Department of Mathematics, University of A Coruña. Campus Elviña s/n, 15071 - A Coruña, Spain(mcalvog@udc.es)

‡Department of Mathematics, University of Bologna. Piazza de Porta San Donato 5, Bologna, Italy (andrea.pascucci@unibo.it)

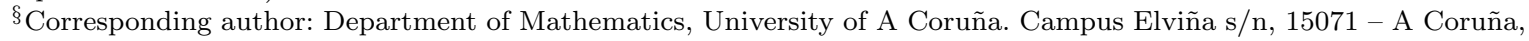
Spain (carlosv@udc.es)
} 
option pricing can be adapted to state a PDE model in the same way as for Asian options with American style. In [20], different models are stated for pension plans depending on the salary at retirement or on the average salary by using a risk neutral probability approach in case without early retirement. More recently, in [13] and [8] the case of early retirement when the benefit of the plan depends on salary at retirement is rigorously analyzed and existence, uniqueness and the properties of the optimal retirement boundary are obtained. In this paper we address the more complex case with retirement payments based on the average salary, which is just posed in [13]. In this case the Black-Scholes model contains two spatial-like variables corresponding to two underlying stochastic factors instead of one.

In the present paper we first decompose the problem into two time regions: one corresponding to the time range to average the salary and the other previous to the initial averaging time. For the first time region, the existence of solution is proved by using the methodology developed in [17] and [16] to study obstacle problems associated to hypoelliptic equations of Kolmogorov type. The regularity of the solution is also analyzed. In the second time region a classical one factor Black-Scholes equation is posed, involving a non-homogeneous term.

Moreover, we provide a numerical method for the PDE discretization by using the techniques developed in [3] for Asian options pricing models. The numerical analysis of the proposed characteristics CrankNicolson time discretization, the fully discretized problem when combined with Lagrange finite elements and the use of numerical integration formulas has been addressed in $[1,2]$. Both papers are applied to general (possibly degenerated) convection-diffusion-reaction equations under certain assumptions. Moreover, the non-linearities associated to the inequality constraints in the complementarity formulation due to early retirement are treated by means of the recently introduced augmented Lagrangian active set method [14]. Among the different possible alternatives (projected relaxation, penalization, other duality techniques...) this method results to be efficient and robust (see, for example, [4] for a comparison with a duality algorithm in an Asian option pricing problem of American style).

The paper is organized as follows. In Section 2 all concerns about the mathematical model are posed. In Section 3 the mathematical analysis tools allows to study the existence and regularity of solution. Section 4 contains the description of the different involved numerical techniques. In Section 5 some examples are presented to illustrate the performance of the proposed numerical method and the behavior of the solution and the optimal retirement boundary.

2. Mathematical modeling. We denote the age of entry of a member in the pension plan by $t_{0}$ and the time since the entry by $t$, so that $t=0$ corresponds to the recruitment date of a person aged $t_{0}$. As the pension plan is indexed to the salary of the member, let us denote by $S_{t_{0}, t}$ (or simply by $S_{t}$ ) the wage at time $t$ of a member entered the pension plan with an age $t_{0}$. Following [20], we assume that $S_{t}$ is governed by the stochastic differential equation

$$
d S_{t}=\alpha\left(t, S_{t}\right) d t+\sigma\left(t, S_{t}\right) d Z_{t}
$$

where:

- the salary growth rate $\alpha$ depends on the time $t$ since the entry into the plan, the current salary $S_{t}$ and the age at entry $t_{0}$

- $\sigma$ denotes the volatility of the salary,

- $Z$ is a Wiener process.

Notice that the model assumes that uncertainty on the salary only depends on the volatility and follows a diffusion model although in real situations some sudden events could produce abrupt changes in the salary. In that case, a jump-diffusion model results to be more appropriate, as discussed in [7].

In this paper we consider a pension plan indicated to average salary during certain number of years before retirement date. Moreover, we incorporate the option of early retirement with a certain penalization. 
So, let us denote by $v_{t}=V\left(t, S_{t} ; t_{0}\right)$ the value at time $t$ of the benefits payable to the member of the plan when he/she is aged $t_{0}+t$ and the salary is $S_{t}$.

In this section we pose the mathematical model in terms of a complementarity problem associated to a PDE to obtain $V$, when the retirement benefits depend on the continuous arithmetic average of the salary during last $n_{y}$ years before retirement date $T_{r}$. Additionally, the early retirement after a given date $T_{0}$, such that $T_{r}-n_{y}<T_{0}<T_{r}$, is allowed. Early retirement option has been analyzed in [13] for the plans indexed to the final salary.

Moreover, it is also assumed the existence of payment of benefits from the fund in case of death of a member or canceling the plan (withdrawal) to move to another plan. Therefore, we assume three possible states of a member of the plan: active $(a)$, death $(d)$ and withdrawal $(w)$. It is considered that retirement occurs at the final age of service in the pension fund unless the involved early retirement option is exercised. The transition intensities from active membership to death or cancellation are denoted by $\mu_{d}\left(t ; t_{0}\right)$ and $\mu_{w}\left(t ; t_{0}\right)$, respectively. In the actuarial approach these intensities are understood as forces of decrement acting at time $t$ on a member aged $t_{0}+t$ and can be expressed in terms of the corresponding transition probabilities from one state to another.

By using actuarial arguments, when assuming deterministic benefits paid to the fund and ignoring any contributions, the variation of the value of the retirement benefits is given by the following Thiele's differential equation (see [6], for example):

$$
d v_{t}=r(t) v_{t} d t-\sum_{i=d, w} \mu_{i}\left(t ; t_{0}\right)\left(A_{i}\left(t, S_{t} ; t_{0}\right)-v_{t}\right) d t
$$

where $r(t)$ is the deterministic time dependent risk-free interest rate and $A_{i}\left(t, S ; t_{0}\right)$ denotes the deterministic benefit paid by the fund in case of death $(i=d)$ or withdrawal $(i=w)$. Notice that the difference $A_{i}\left(t, S_{t} ; t_{0}\right)-v_{t}$ represents the sum-at-risk associated to the corresponding decrement $i$, so that

$$
\sum_{i=d, w} \mu_{i}\left(t ; t_{0}\right)\left(A_{i}\left(t, S_{t} ; t_{0}\right)-v_{t}\right) d t
$$

denotes the instantaneous expected value of the payments from the fund.

Following Section 5 in [20], we assume that

$$
A_{i}\left(t, S ; t_{0}\right)=\alpha_{i} S, \quad \alpha_{i} \geq 0, \quad i=d, w,
$$

so that the death and withdrawal benefits are a constant multiple of the salary, and that the transition intensities $\mu_{i}\left(t_{0} ; t\right)=\mu_{i}$ are nonnegative constants.

As we are considering that retirement benefits depend on the continuous arithmetic average of the salary, analogously to the case of Asian options, we introduce the following variable representing the cumulative function of the salary since the last $n_{y}$ years before $T_{r}$ :

$$
I_{t}=\int_{0}^{t} g\left(\tau, S_{\tau}\right) d \tau
$$

with

$$
g(t, S)=\left\{\begin{array}{lll}
0 & \text { if } & 0 \leq t<T_{r}-n_{y} \\
h(S) & \text { if } & T_{r}-n_{y} \leq t \leq T_{r}
\end{array}\right.
$$

where $h$ is appropriately chosen. Specifically, in this paper we consider the particular case $h(S)=k_{1} S$, where the accrual constant $k_{1}$ is positive, that is

$$
k_{1}>0
$$


For simplicity, hereafter we drop the dependence on the entry age " $t_{0}$ " in all functions (in particular, the value of the plan is denoted by $\left.v_{t}=V\left(t, S_{t}, I_{t} ; t_{0}\right)=V\left(t, S_{t}, I_{t}\right)\right)$. Then we can apply Ito lemma to $v_{t}$ jointly with Thiele differential equation (2.1) to obtain

$$
\begin{aligned}
d v_{t}= & \left(\partial_{t} V\left(t, S_{t}, I_{t}\right)+\alpha\left(t, S_{t}\right) \partial_{S} V\left(t, S_{t}, I_{t}\right)+g\left(t, S_{t}\right) \partial_{I} V\left(t, S_{t}, I_{t}\right)+\frac{\sigma\left(t, S_{t}\right)^{2}}{2} \partial_{S S} V\left(t, S_{t}, I_{t}\right)\right) d t \\
& +\sigma\left(t, S_{t}\right) \partial_{S} V\left(t, S_{t}, I_{t}\right) d Z_{t}+\sum_{i=d, w} \mu_{i}\left(A_{i}\left(t_{0}, t, S_{t}\right)-v_{t}\right) d t
\end{aligned}
$$

where the first two terms on the right hand side are associated to the stochastic variation of the salary while the third term is related to the expected payments due to death or withdrawal.

In [20] the PDE for the case without early retirement is obtained by arguing that the risk-adjusted expected change in the liabilities value, after allowing the benefits cash flows from the fund, should be equal to the risk-free interest rate. More recently, in [7] a dynamic hedging methodology to deduce the PDE for the same case without early retirement option is applied. In both papers the following PDE for the value of the benefits of the pension plan

$$
\partial_{t} V+\beta \partial_{S} V+g \partial_{I} V+\frac{\sigma^{2}}{2} \partial_{S S} V-\left(\mu_{d}+\mu_{w}+r\right) V=-\mu_{d} A_{d}-\mu_{w} A_{w},
$$

is obtained, where $\beta=\alpha-\lambda \sigma$ is related to the market price of risk associated to the uncertainty of the salary, here denoted by $\lambda$. Assuming that at the retirement date $T_{r}$ the owner of the pension plan receives a fraction of the average salary during last $n_{y}$ years, the pension plan pricing model for the case without early retirement is defined by equation (2.6) jointly with the final condition

$$
V\left(T_{r}, S, I\right)=\frac{a I}{n_{y}}
$$

where $a \in] 0,1[$ is a given accrual constant.

In [7] the existence, uniqueness and regularity of the solution are analyzed. Moreover, the proposed numerical method to solve the PDE problem (2.6)-(2.7) and some numerical examples are described.

If early retirement is allowed, it seems reasonable to assume that a member of the plan would retire when he/she maximizes the benefits of retirement among all possible dates (optimal stopping time) to retire. The financial argument results to be very similar to the one used when pricing financial products including the option of early exercise (American options, callable bonds, Bermudan products,...). On the other hand, from the pension plan manager viewpoint it seems reasonable to penalize the benefits received by the member when he/she decides to retire before the expected retirement date. Thus, we assume that the member will receive at the early retirement date $t$, the quantity given by

$$
\Psi(t, S, I)= \begin{cases}0 & \text { if } t<T_{0} \\ \left(1-\frac{T_{r}-t}{T_{r}-T_{0}}\right) \frac{a I}{t-\left(T_{r}-n_{y}\right)} & \text { if } t \geq T_{0} .\end{cases}
$$

Notice that retirement is not allowed before $T_{0}$ so that we assume the member receives nothing when retiring before that date. Then, the value of the pension plan benefits can be expressed as

$$
V\left(t, S_{t}, I_{t}\right)=\sup _{\tau \in \mathcal{T}(t, T)} E\left[e^{-r(\tau-t)} \Psi\left(\tau, S_{\tau}, I_{\tau}\right)\right]
$$

where $\mathcal{T}(t, T)$ denotes the set of stopping times between $t$ and $T$. 
By using analogous arbitrage free arguments to the ones argued in early exercise financial products, the related PDE problem can be written in terms of a complementarity problem as follows:

$$
\begin{cases}\max \{\mathcal{L} V-f, \Psi-V\}=0, & \text { in }] 0, T_{r}\left[\times \mathbb{R}_{+}^{2},\right. \\ V\left(T_{r}, S, I\right)=\frac{a I}{n_{y}}, & (S, I) \in \mathbb{R}_{+}^{2}\end{cases}
$$

where

$$
\begin{aligned}
& \mathcal{L} V=\partial_{t} V+\beta \partial_{S} V+g \partial_{I} V+\frac{\sigma^{2} S^{2}}{2} \partial_{S S} V-\left(r+\mu_{d}+\mu_{w}\right) V, \\
& f(t, S, I)=-\left(\mu_{d} \alpha_{d}+\mu_{w} \alpha_{w}\right) S .
\end{aligned}
$$

3. Existence results. In the previous section, the mathematical model for the benefit of the pension plan with the early retirement option has been posed as the Cauchy linear complementarity problem (2.10). In this section we study the existence and regularity of solutions.

In what follows we assume that $\sigma$ and $\beta$ are proportional to the salary, so that $\sigma(t, S)=\sigma S$ and $\beta(t, S, I)=\theta S$, where $\theta>0$ and $\sigma>0$ are given constants. The assumption on $\sigma$ implies that the salary volatility increases with salary, which is a reasonable argument, mainly in the private sector. Additionally, the joint assumption on the expressions of both $\sigma$ and $\beta$ implies that the market price of risk is a constant parameter when a log-normal evolution for salaries is considered (that is, when $\alpha(t, S)=\alpha S)$.

Next, having in view that the function $g$ in (2.4) vanishes when $t<T_{r}-n_{y}$, in order to analyze the existence of solutions, we decompose (2.10) into two different problems:

\section{- Problem 1:}

Find a function $V_{1}$, defined in $\left[T_{r}-n_{y}, T_{r}\right] \times \mathbb{R}_{+}^{2}$, such that

$$
\begin{cases}\max \left\{\mathcal{L}_{1} V_{1}-f, \Psi-V_{1}\right\}=0, & \text { in }] T_{r}-n_{y}, T_{r}\left[\times \mathbb{R}_{+}^{2},\right. \\ V_{1}\left(T_{r}, S, I\right)=\frac{a I}{n_{y}}, & (S, I) \in \mathbb{R}_{+}^{2}\end{cases}
$$

where

$$
\mathcal{L}_{1} V=\partial_{t} V+\theta S \partial_{S} V+k_{1} S \partial_{I} V+\frac{\sigma^{2} S^{2}}{2} \partial_{S S} V-k_{2} V, \quad f(t, S, I)=-k_{3} S
$$

with

$$
k_{2}=r+\mu_{d}+\mu_{w}, \quad k_{3}=\mu_{d} \alpha_{d}+\mu_{w} \alpha_{w} .
$$

\section{- Problem 2:}

For each $I>0$, find a function $V_{2}(\cdot, \cdot ; I)$ defined in $\left[0, T_{r}-n_{y}\right] \times \mathbb{R}_{+}$, such that

$$
\begin{cases}\mathcal{L}_{2} V_{2}(\cdot, \cdot ; I)=f, & \text { in }] 0, T_{r}-n_{y}\left[\times \mathbb{R}_{+},\right. \\ V_{2}\left(T_{r}-n_{y}, S ; I\right)=V_{1}\left(T_{r}-n_{y}, S, I\right), & S \in \mathbb{R}_{+},\end{cases}
$$

where

$$
\mathcal{L}_{2} V=\partial_{t} V+\theta S \partial_{S} V+\frac{\sigma^{2} S^{2}}{2} \partial_{S S} V-k_{2} V
$$

and $f$ and $k_{2}$ are as in (3.2)-(3.3). 
Problem 1 consists of a complementarity problem associated to the degenerate parabolic operator $\mathcal{L}_{1}$. Notice that the obstacle function $\Psi$ vanishes for $t \leq T_{0}$ and therefore in this time range the inequality constraint reduces to the positivity of the solution, which is in turn guaranteed by the positivity of the final condition and the maximum principle. Problem 2 consists of a parameterized family of onedimensional Cauchy problems (associated to the standard non-homogeneous Black-Scholes equation with linear second member) depending on $I$ as a parameter through the final condition.

In the next subsections we discuss the existence of solutions for both problems.

3.1. Mathematical analysis of Problem 1.. We refer to operator $\mathcal{L}_{1}$ in Problem 1 as an ultraparabolic operator because only the first order derivative w.r.t. $I$ appears, while the solution is a function of the spatial variables $S, I$ and the time variable $t$. Recently, in the study of the arithmetic American Asian option pricing problem, Monti and Pascucci in [16] proved the existence and uniqueness of the solution of a homogeneous problem which is similar to Problem 1. In this section, we generalize those results to include (3.1).

As a first step, we construct a supersolution for (3.1).

DeFinition 3.1. A function $\left.\left.\bar{U} \in C^{2}(] T_{r}-n y, T_{r}\left[\times \mathbb{R}_{+}^{2}\right) \cap C(] T_{r}-n y, T_{r}\right] \times \mathbb{R}_{+}^{2}\right)$ such that

$$
\left.\mathcal{L}_{1} \bar{U} \leq f \quad \text { and } \quad \bar{U} \geq \Psi \quad \text { in }\right] T_{r}-n_{y}, T_{r}\left[\times \mathbb{R}_{+}^{2},\right.
$$

is called a supersolution to problem (3.1). We can obtain a super-solution in two different ways: either we perform the same changes of variables that in [7] or we work in the original variables and try to obtain the same kind of supersolution used in [16]. In the present paper we follow the latter approach.

Proposition 3.2. If $\gamma$ and $\delta$ are suitably large constants then the function $\bar{U}$ defined by

$$
\bar{U}(t, S, I)=\gamma e^{-\delta t}\left(S+\sqrt{S^{2}+I^{2}}\right)
$$

is a super-solution to problem (3.1).

Proof. We set

$$
\bar{U}=\bar{U}_{1}+\bar{U}_{2},
$$

where

$$
\bar{U}_{1}(t, S, I)=\gamma e^{-\delta t} \sqrt{S^{2}+I^{2}} \quad \text { and } \quad \bar{U}_{2}(t, S, I)=\gamma e^{-\delta t} S .
$$

Recalling that $f(t, S, I)=-k_{3} S$, the thesis follows from the following points:

i) there exists $\delta_{0}>0$ such that

$$
\mathcal{L}_{1} \bar{U}_{1} \leq 0
$$

for any $\gamma \geq 0$ and $\delta \geq \delta_{0}$. Indeed we have

$$
\mathcal{L}_{1} \bar{U}_{1}(t, S, I)=\frac{\gamma e^{-t \delta}}{2\left(I^{2}+S^{2}\right)^{3 / 2}} W(S, I)
$$

where

$$
W(S, I):=\sigma^{2} S^{2} I^{2}-2\left(I^{2}+S^{2}\right)\left(S^{2}\left(k_{2}+\delta-\theta\right)+I^{2}\left(k_{2}+\delta\right)-k_{1} I S\right) .
$$


Therefore $\mathcal{L}_{1} \bar{U}_{1} \leq 0$ if and only if $W(S, I) \leq 0$. By the elementary inequality

$$
|S I| \leq \frac{S^{2}+I^{2}}{2}
$$

we have

$$
W(S, I) \leq\left(\frac{S^{2}+I^{2}}{2}\right)^{2} \sigma^{2}-2\left(S^{2}+I^{2}\right)\left(\left(k_{1}+k_{2}+\delta-\theta\right) S^{2}+\left(k_{1}+k_{2}+\delta\right) I^{2}\right) .
$$

Therefore $W(S, I) \leq 0$ if and only if

$$
\frac{S^{2}+I^{2}}{4} \sigma^{2}-2\left(\left(k_{1}+k_{2}+\delta-\theta\right) S^{2}+\left(k_{1}+k_{2}+\delta\right) I^{2}\right) \leq 0,
$$

which is true if $\delta$ is sufficiently large;

ii) there exists $\gamma_{0}$ such that

$$
\left.\mathcal{L}_{1} \bar{U}_{2}(t, S, I)+k_{3} S \leq 0, \quad(t, S, I) \in\right] T_{r}-n_{y}, T_{r}\left[\times \mathbb{R}_{+}^{2},\right.
$$

for any $\gamma \geq \gamma_{0}$ and $\delta \geq \max \left\{\delta_{0}, \theta-k_{2}\right\}$. More precisely, inequality (3.9) follows from the identity

$$
\mathcal{L}_{1} \bar{U}_{2}(t, S, I)+k_{3} S=\left(\gamma e^{-\delta t}\left(\theta-k_{2}-\delta\right)+k_{3}\right) S ;
$$

iii) finally, for any $\delta \geq 0$ there exists $\gamma \geq \gamma_{0}$ such that

$$
\bar{U}(t, S, I) \geq \gamma e^{-\delta T_{r}} I \geq \Psi(t, S, I), \quad(t, S, I) \in\left[T_{r}-n_{y}, T_{r}\right] \times \mathbb{R}_{+}^{2} .
$$

Existence and regularity of solutions to (3.1) are more delicate matters. Indeed, on the one hand operator $\mathcal{L}_{1}$ is not uniformly parabolic so the classical theory does not apply; on the other hand, it is wellknown that even in the standard case of uniformly parabolic operators, a complementarity problem does not admit classical solutions. Following [16], we study problem (3.1) in the framework of hypoelliptic equations of Kolmogorov type. More precisely, we consider an operator in $\mathbb{R}^{3}$ of the form

$$
L=\bar{a}(t, S, I) \partial_{S S}+Y,
$$

where

$$
Y=\partial_{t}+\theta S \partial_{S}+k_{1} S \partial_{I}
$$

is the first order part of $\mathcal{L}_{1}$. By the results in [19] and [9], it is known that under the assumption (2.5) (i.e. $k_{1}>0$ ) and if the coefficient $\bar{a}$ is a smooth function such that

$$
\frac{1}{\mu} \leq \bar{a} \leq \mu \quad \text { on } \mathbb{R}^{3},
$$

for some positive constant $\mu$, then operator $L$ has a global fundamental solution which can be estimated by Gaussian functions. Notice that $\mathcal{L}_{1}$ is locally of the form $(3.10)$ since the function $\bar{a}(t, S, I)=\frac{\sigma^{2} S^{2}}{2}$ verifies the non-degeneracy condition (3.12) on compact subsets of $\mathbb{R} \times \mathbb{R}_{+}^{2}$.

The obstacle problem for a general class of degenerate parabolic operators including (3.10) was first studied by Di Francesco, Pascucci and Polidoro in [10] who proved the existence of strong solutions: specifically, for any domain $\Omega$ of $\mathbb{R}^{3}$ and $p \geq 1$, we introduce the Sobolev-Stein spaces

$$
\mathcal{S}^{p}(\Omega)=\left\{U \in L^{p}(\Omega) \mid \partial_{S} U, \partial_{S S} U, Y U \in L^{p}(\Omega)\right\}
$$


endowed with the semi-norm

$$
\|U\|_{\mathcal{S}^{p}}=\|U\|_{L^{p}}+\left\|\partial_{S} U\right\|_{L^{p}}+\left\|\partial_{S S} U\right\|_{L^{p}}+\|Y U\|_{L^{p}} .
$$

If $u \in \mathcal{S}^{p}\left(\Omega^{\prime}\right)$ for any compact subset $\Omega^{\prime} \subseteq \Omega$, then we write $\mathcal{S}_{\text {loc }}^{p}(\Omega)$.

Definition 3.3. A strong solution to problem (3.1) is a function $\left.U \in \mathcal{S}_{\text {loc }}^{1} \cap C(] T_{r}-n_{y}, T_{r}\right] \times \mathbb{R}_{+}^{2}$ ) which satisfies the differential inequality a.e. in $] T_{r}-n_{y}, T_{r}\left[\times \mathbb{R}_{+}^{2}\right.$ and the final condition in the pointwise sense. The main result of this section in the following

TheOrem 3.4. Problem (3.1) admits a strong solution $U$ which belongs to $\mathcal{S}_{\mathrm{loc}}^{p}$ for any $p \geq 1$ and satisfies the inequality

$$
U \leq \bar{U}
$$

where $\bar{U}$ is the supersolution in (3.7).

Proof. Let $D_{\rho}\left(x_{1}, x_{2}\right)$ denote the Euclidean ball centered at $\left(x_{1}, x_{2}\right) \in \mathbb{R}^{2}$, with radius $\rho$. We consider the sequence of domains $O_{n}=D_{n}\left(n+\frac{1}{n}, 0\right) \cap D_{n}\left(0, n+\frac{1}{n}\right)$ covering $\mathbb{R}_{+}^{2}$. For any $n \in \mathbb{N}$, the cylinder $\left.H_{n}=\right] T_{r}-n_{y}, T_{r}\left[\times O_{n}\right.$ is a $\mathcal{L}_{1}$-regular domain in the sense that there exists a barrier function at any point of the parabolic boundary $\partial_{P} H_{n}:=\partial H_{n} \backslash\left(\{0\} \times O_{n}\right)$ (cf. Remark 3.1 in [10]). Since $\mathcal{L}_{1}$ satisfies condition (3.12) on $H_{n}$ for any $n$, then by Theorem 3.1 in [10] we have: for any $n \in \mathbb{N}$, there exists a strong solution $U_{n} \in \mathcal{S}_{\text {loc }}^{p}\left(H_{n}\right) \cap C\left(H_{n} \cup \partial_{P} H_{n}\right)$ to problem

$$
\left\{\begin{array}{l}
\max \left\{\mathcal{L}_{1} U-f, \Psi-U\right\}=0 \quad \text { in } H_{n}, \\
\left.u\right|_{\partial_{P} H_{n}}=\frac{a I}{n_{y}} .
\end{array}\right.
$$

Moreover, the following estimate holds: for every $p \geq 1$ and $H \subset \subset H_{n}$ there exists a positive constant $C$, only depending on $H, H_{n}, p,\|\Psi\|_{L^{\infty}\left(H_{n}\right)}$ such that

$$
\left\|U_{n}\right\|_{\mathcal{S}^{p}(H)} \leq C
$$

Next we consider a sequence of cut-off functions $\chi_{n} \in C_{0}^{\infty}\left(\mathbb{R}_{+}^{2}\right)$, such that $\chi_{n}=1$ on $O_{n-1}, \chi_{n}=0$ on $\mathbb{R}_{+}^{2} \backslash O_{n}$ and $0 \leq \chi_{n} \leq 1$. We set

$$
\Psi_{n}(t, S, I)=\chi_{n}(S, I) \Psi(t, S, I)+\left(1-\chi_{n}(S, I)\right) \bar{U}(t, S, I),
$$

where $\bar{U}$ is the supersolution in (3.7), and we denote by $U_{n}$ the strong solution to (3.14) with $\Psi=\Psi_{n}$. By the comparison principle we have $\Psi \leq U_{n} \leq U_{n+1} \leq \bar{U}$ and therefore, by estimate (3.15), for every $p \geq 1$ and $H \subset \subset H_{n}$ we have $\left\|U_{n}\right\|_{\mathcal{S}^{p}(H)} \leq C$ for some constant $C$ depending on $H$ but not on $n$. Then we can pass to the limit as $n \rightarrow \infty$, on compact subsets of $] T_{r}-n_{y}, T_{r}\left[\times \mathbb{R}_{+}^{2}\right.$, to get a strong solution of $\max \left\{\mathcal{L}_{1} U-f, \Psi-U\right\}=0$. A standard argument based on barrier functions shows that $U(t, \cdot)$ is continuous up to $t=T_{r}$ and attains the final datum.

Next, we briefly discuss the regularity properties of the strong solution $U$ of problem (3.1). For greater convenience, we put $x=(S, I)$ and, using the matrix notation, we rewrite the vector field $Y$ in (3.11) as

$$
Y=\left\langle B x, \nabla_{x}\right\rangle+\partial_{t}
$$

where

$$
B=\left(\begin{array}{cc}
\theta & 0 \\
k_{1} & 0
\end{array}\right)
$$


and $\nabla_{x}$ is the gradient in the variables $x$. As observed by Lanconelli and Polidoro [15], in the case that the coefficient $\bar{a}$ in (3.10) is constant, then operator $L$ is invariant ${ }^{1}$ w.r.t. the left translations in the group law

$$
(\tau, \xi) *(t, x)=\left(\tau+t, x+e^{t B} \xi\right)
$$

where

$$
e^{t B}=\left(\begin{array}{cc}
e^{t \theta} & 0 \\
\frac{k_{1}\left(e^{t \theta}-1\right)}{\theta} & 1
\end{array}\right) .
$$

In addition, if $\theta=0$ then $L$ is also homogeneous of degree two ${ }^{2}$ w.r.t the dilations group

$$
\delta_{\lambda}(t, S, I)=\left(\lambda^{2} t, \lambda S, \lambda^{3} I\right), \quad \lambda>0 .
$$

It turns out that the operations just defined are naturally designed for the study of the optimal regularity properties of the solution in Theorem 3.4. Indeed, let us first recall the following embedding theorem proved in [10].

TheOREM 3.5. Let $O, \Omega$ be bounded domains of $\mathbb{R}^{3}$ such that $O \subset \subset \Omega$ and $p>8$. There exists a positive constant $c$, only dependent on $B, \Omega, O$ and $p$, such that

$$
\|u\|_{C_{B}^{1, \alpha}(O)} \leq c\|u\|_{\mathcal{S}^{p}(\Omega)}, \quad \alpha=1-\frac{8}{p},
$$

for any $u \in \mathcal{S}^{p}(\Omega)$. In (3.16), the anisotropic Hölder space $C_{B}^{1, \alpha}$ is defined in terms of the following norms ${ }^{3}$ :

$$
\begin{aligned}
& \|U\|_{C_{B}^{0, \alpha}(\Omega)}=\sup _{\Omega}|U|+\sup _{\substack{(t, x),(\tau, \xi) \in \Omega \\
(t, x) \neq(\tau, \xi)}} \frac{|U(t, x)-U(\tau, \xi)|}{\left\|(\tau, \xi)^{-1} *(t, x)\right\|_{B}^{\alpha}}, \\
& \|U\|_{C_{B}^{1, \alpha}(\Omega)}=\|U\|_{C_{B}^{0, \alpha}(\Omega)}+\left\|\partial_{S} U\right\|_{C_{B}^{0, \alpha}(\Omega)}+\sup _{\substack{(t, x),(\tau, \xi) \in \Omega \\
(t, x) \neq(\tau, \xi)}} \frac{\left|U(t, x)-U(\tau, \xi)-\left(S-S^{\prime}\right) \partial_{S} U(\tau, \xi)\right|}{\left\|(\tau, \xi)^{-1} *(t, x)\right\|_{B}^{1+\alpha}},
\end{aligned}
$$

where $\|\cdot\|_{B}$ is the $\delta_{\lambda}$-homogeneous norm in $\mathbb{R}^{3}$ defined by

$$
\|(t, S, I)\|_{B}=|t|^{\frac{1}{2}}+|S|+|I|^{\frac{1}{3}} .
$$

As a consequence of Theorem 3.5, the strong solution of problem (3.1) belongs locally to the space $C_{B}^{1, \alpha}$ for any $\alpha<1$. Actually, sharp interior regularity results for a class of problems including (3.1) were proved in [12]: it turns out that the solution of (3.1) belongs to the class $\mathcal{S}_{\text {loc }}^{\infty}$ and this is the optimal regularity for this kind of problem.

${ }^{1} L$ is left- $*$-invariant if

$$
L U((\tau, \xi) *(t, x))=(L U)((\tau, \xi) *(t, x)) .
$$

${ }^{2} L$ is $\delta_{\lambda}$-homogeneous of degree two if

$$
L U\left(\delta_{\lambda}(t, x)\right)=\lambda^{2}(L U)\left(\delta_{\lambda}(t, x)\right) .
$$

${ }^{3}$ We use the notation $x=(S, I)$ and $\xi=\left(S^{\prime}, I^{\prime}\right)$. 
We remark explicitly that, for any bounded domain $\Omega$, there exists a positive constant $c_{\Omega}$ such that

$$
\begin{aligned}
\left\|(\tau, \xi)^{-1} *(t, x)\right\|_{B} & =\left\|(t-\tau, x-\xi)+\left(0,\left(\operatorname{Id}_{2}-e^{(t-\tau) B}\right) \xi\right)\right\|_{B} \\
& \leq c_{\Omega}|(t-\tau, x-\xi)|^{\frac{1}{3}}, \quad(\tau, \xi),(t, x) \in \Omega,
\end{aligned}
$$

where $\operatorname{Id}_{2}$ is the identity matrix in $\mathbb{R}^{2}$. In particular, we have

$$
C_{B}^{0, \alpha}(\Omega) \subseteq C^{0, \frac{\alpha}{3}}(\Omega)
$$

where $C^{0, \alpha}$ denotes the standard Euclidean Hölder space.

Moreover, if $U \in C_{B}^{1, \alpha}(\Omega)$ then $U, \partial_{S} U \in C_{B}^{0, \alpha}(\Omega)$ and also

$$
|U((t, x) *(\tau, 0))-U(t, x)|=\left|U\left(\left(t+\tau, e^{\tau B} x\right)\right)-U(t, x)\right| \leq c_{\Omega}|\tau|^{\frac{1+\alpha}{2}}
$$

which implies the Hölder regularity of order $\frac{1+\alpha}{2}$ along the integral curves of $Y$. As a matter of fact, $Y$ can be identified with the vector field $Y(t, x)=(1, B x)$ and $\gamma(\tau):=\left(t+\tau, e^{\tau B} x\right)$ is the integral curve of $Y$ starting from $(t, x)$, that is the solution of the problem

$$
\frac{d \gamma(\tau)}{d \tau}=Y(\gamma(\tau)), \quad \gamma(0)=(t, x)
$$

Notice however that the $C_{B}^{1, \alpha}$-regularity of $U$ does not imply the existence of the Euclidean derivative $\partial_{I} U$ : roughly speaking, $\partial_{I}$ can be recovered by commuting $\partial_{S}$ and $Y$

$$
\left[\partial_{S}, Y\right]=\partial_{S} Y-Y \partial_{S}=\theta \partial_{S}+k_{1} \partial_{I}
$$

and therefore intrinsically it has to be considered as a third order derivative. For this reason, in the numerical solution of the problem we adopt the natural approach of using a semi-Lagrangian method for time discretization, that mainly consists of a finite differences scheme along the integral curves of the convective part $Y$ of the equation (cf. Subsection 4.1).

Concerning Problem 2, up to a logarithmic change of variable, it consists of a Cauchy problem for a nonhomogeneous heat equation with a continuous final datum which, by (3.13), has exponential growth at infinity. Thus, standard results guarantee existence and uniqueness of a classical solution $V_{2}$. Moreover, continuous dependence results imply that $V_{2}$ inherits the regularity in the $I$ variable from the final datum.

4. Numerical methods. In our numerical approach, the methods have been designed to solve the problem without distinguishing the time region where actually only one factor is acting from the region where the PDE is governed by the two stochastic factors (Problem 1). Thus, the numerical solution of the complementarity problem formulation (2.10) is addressed. Although this approach involves a bit more computational cost it simplifies the computational code.

In order to enumerate the numerical techniques, the main difficulties and the way to overcome them numerically are briefly outlined. First, as in the case without early exercise opportunity, a localization technique is used to cope with the initial formulation in an unbounded domain. Also, as the diffusive term is strongly degenerate so that the PDE can be understood as an example of extreme convective dominated case, we propose a Crank-Nicolson characteristics time discretization scheme combined with piecewise quadratic Lagrange finite element method. For the inequality constraints associated to the early retirement option, we propose a mixed formulation and the use of an augmented Lagrangian active set technique. 
Therefore, we first consider a problem posed in a sufficiently large spatial bounded domain, so that the solution in the region of financial interest is not affected by the truncation of the unbounded domain and the required boundary conditions (localization procedure). For this purpose, as in the case without early retirement treated in [7], we first introduce the change of variable in time and notation

$$
\tau=T_{r}-t, \quad x_{1}=S, \quad x_{2}=I .
$$

Let us consider $x_{1}^{\infty}$ and $x_{2}^{\infty}$ be large enough real numbers and let $\left.\Omega=\right] 0, x_{1}^{\infty}[\times] 0, x_{2}^{\infty}$ [ be the computational bounded domain. Moreover, let $\partial \Omega=\bigcup_{i=1}^{2}\left(\Gamma_{i}^{-} \bigcup \Gamma_{i}^{+}\right)$, with $\Gamma_{i}^{-}=\left\{\left(x_{1}, x_{2}\right) \in \partial \Omega \mid x_{i}=0\right\}$ and $\Gamma_{i}^{+}=\left\{\left(x_{1}, x_{2}\right) \in \partial \Omega \mid x_{i}=x_{i}^{\infty}\right\}$ for $i=1,2$.

The iterative algorithms we propose for the numerical solution of (2.10) are based on the Lagrangian formulation of the complementarity problem. This approach mainly consists on replacing the inequality in (2.10) by an identity in terms of an appropriate Lagrange variable or multiplier to be denoted by $P$. This mixed formulation classically appears when dealing with duality methods for solving obstacle problems (see [11], for example). Then, problem (2.10) admits an equivalent mixed formulation. Moreover, after the domain truncation, this mixed formulation in the unbounded domain can be approximated by the following one in the bounded domain:

Find $V$ and $P:\left[0, T_{r}\right] \times \Omega \longrightarrow R$, satisfying the partial differential equation

$$
\left.V_{\tau}-\operatorname{div}(A \nabla V)+\vec{v} \cdot \nabla V+l V+P=f \quad \text { in }\right] 0, T_{r}[\times \Omega,
$$

the complementarity conditions

$$
V \geq \bar{\Psi}, \quad P \leq 0, \quad(V-\bar{\Psi}) P=0 \quad \text { in }] 0, T_{r}[\times \Omega,
$$

the initial condition

$$
V\left(0, x_{1}, x_{2}\right)=\frac{a x_{2}}{n_{y}} \quad \text { in } \Omega
$$

and the boundary conditions

$$
\begin{aligned}
& \frac{\partial V}{\partial x_{1}}=g_{1} \text { on }\left(0, T_{r}\right) \times \Gamma_{1}^{+}, \\
& \frac{\partial V}{\partial x_{2}}=g_{2} \text { on }\left(0, T_{r}\right) \times \Gamma_{2}^{+},
\end{aligned}
$$

where the involved data are defined as follows

$$
\begin{aligned}
& A\left(x_{1}, x_{2}\right)=\left(\begin{array}{cc}
\frac{1}{2} \sigma^{2} x_{1}^{2} & 0 \\
0 & 0
\end{array}\right), \quad \vec{v}\left(\tau, x_{1}, x_{2}\right)=\left(\begin{array}{c}
\left(\sigma^{2}-\theta\right) x_{1} \\
-g\left(T_{r}-\tau, x_{1}\right)
\end{array}\right), \\
& l\left(\tau, x_{1}, x_{2}\right)=r+\mu_{d}+\mu_{w}, \quad f\left(\tau, x_{1}, x_{2}\right)=\left(\mu_{d} \alpha_{d}+\mu_{w} \alpha_{w}\right) x_{1}, \\
& \varphi\left(x_{1}, x_{2}\right)=\frac{a}{n_{y}} x_{2}, \quad \bar{\Psi}\left(\tau, x_{1}, x_{2}\right)=\Psi\left(T_{r}-\tau, x_{1}, x_{2}\right) \\
& \quad g_{1}\left(\tau, x_{1}, x_{2}\right)=0, \quad g_{2}\left(\tau, x_{1}, x_{2}\right)=\frac{a}{n_{y}} .
\end{aligned}
$$

Note that the presence of the boundary conditions at the new boundaries of the bounded domain are related to the expression of the velocity field and the diffusion matrix. This issue has been widely discussed in [7]. 
4.1. Lagrange-Galerkin discretization. Concerning the numerical methods, in the present subsection we mainly describe the solution of the free-boundary aspect related to the early retirement possibility. Nevertheless, we summarize the description of the second order Lagrange-Galerkin method that has been analyzed in [1,2] for time-space discretization. Recently, its application the pension plan model without early retirement is described in detail in [7].

The method of characteristics is based on a finite differences scheme for the discretization of the material derivative, i.e. the time derivative along the characteristic lines of the convective part of the equation [18]. The material derivative operator is given by:

$$
\frac{D}{D \tau}=\partial_{\tau}+\vec{v} \cdot \nabla
$$

For a brief description of the method, we first define the characteristics curve $X(\mathbf{x}, \bar{\tau} ; s)$ through $\mathbf{x}=$ $\left(x_{1}, x_{2}\right)$ at time $\bar{\tau}$, i.e. the integral path associated to the vector field $\vec{v}$ through $\mathbf{x}$, which verifies

$$
\frac{\partial}{\partial s} X(\mathbf{x}, \bar{\tau} ; s)=\vec{v}(X(\mathbf{x}, \bar{\tau} ; s)), \quad X(\mathbf{x}, \bar{\tau} ; \bar{\tau})=\mathbf{x} .
$$

For $N>1$ let us consider the time step $\Delta \tau=T_{r} / N$ and the time mesh-points $\tau_{n}=n \Delta \tau, n=$ $0, \frac{1}{2}, 1, \frac{3}{2}, \ldots, N$. The material derivative approximation by the characteristics method is given by

$$
\frac{D V}{D \tau}=\frac{V^{n+1}-V^{n} \circ X^{n}}{\Delta \tau},
$$

where $X^{n}(\mathbf{x}):=X\left(\mathbf{x}, \tau^{n+1} ; \tau^{n}\right)$. In view of the expression of the velocity field and the continuous function $g$ given by (4.7) and (2.4) respectively, the components of $X^{n}(\mathbf{x})$ can be analytically computed. More precisely, we distinguish the following two cases:

- if $\theta \neq \sigma^{2}$ then $\left[X^{n}\right]_{1}(\mathbf{x})=x_{1} \exp \left(\left(\theta-\sigma^{2}\right) \Delta \tau\right)$ and

$$
\left[X^{n}\right]_{2}(\mathbf{x})=\left\{\begin{array}{lll}
x_{2} & \text { if } & n \Delta \tau>n_{y} \\
\frac{k_{1} x_{1}}{\sigma^{2}-\theta}\left(1-\exp \left(\left(\theta-\sigma^{2}\right) \Delta \tau\right)\right)+x_{2} & \text { if } & n \Delta \tau \leq n_{y}
\end{array}\right.
$$

- if $\theta=\sigma^{2}$ then $\left[X^{n}\right]_{1}(\mathbf{x})=x_{1}$ and

$$
\left[X^{n}\right]_{2}(\mathbf{x})=\left\{\begin{array}{lll}
x_{2} & \text { if } & n \Delta \tau>n_{y} \\
k_{1} x_{1} \Delta \tau+x_{2} & \text { if } & n \Delta \tau \leq n_{y}
\end{array}\right.
$$

Next, we consider a Crank-Nicolson scheme around $\left(X\left(\mathbf{x}, \tau_{n+1} ; \tau\right), \tau\right)$ for $\tau=\tau_{n+\frac{1}{2}}$. So, for $n=$ $0, \ldots, N-1$, the time discretized equation for the case without early retirement $(P=0)$ can be written as:

- Find $V^{n+1}$ such that

$$
\begin{array}{r}
\frac{V^{n+1}(\mathbf{x})-V^{n}\left(X^{n}(\mathbf{x})\right)}{\Delta \tau}-\frac{1}{2} \operatorname{div}\left(A \nabla V^{n+1}\right)(\mathbf{x})-\frac{1}{2} \operatorname{div}\left(A \nabla V^{n}\right)\left(X^{n}(\mathbf{x})\right)+ \\
\frac{1}{2}\left(l V^{n+1}\right)(\mathbf{x})+\frac{1}{2}\left(l V^{n}\right)\left(X^{n}(\mathbf{x})\right)= \\
\frac{1}{2} f^{n+1}(\mathbf{x})+\frac{1}{2} f^{n}\left(X^{n}(\mathbf{x})\right) .
\end{array}
$$


In [7] an appropriate variational formulation for the previously time discretized problem has been stated. Moreover, for the spatial discretization a piecewise quadratic finite elements space can be used. For this purpose, we consider $\left\{\tau_{h}\right\}$ a quadrangular mesh of the domain $\Omega$. Let $\left(T, \mathcal{Q}_{2}, \Sigma_{T}\right)$ be a family of piecewise quadratic Lagrangian finite elements, where $\mathcal{Q}_{2}$ is the space of polynomials defined in $T \in \tau_{h}$ with degree less or equal than two in each spatial variable and $\Sigma_{T}$ denotes the subset of nodes of the element $T$. More precisely, let us define the finite elements space $V_{h}$ by

$$
V_{h}=\left\{\varphi_{h} \in \mathcal{C}^{0}(\bar{\Omega}) \mid \varphi_{h_{T}} \in \mathcal{Q}_{2}, \forall T \in \tau_{h}\right\},
$$

where $\mathcal{C}^{0}(\bar{\Omega})$ is the space of continuous functions on $\bar{\Omega}$. Indeed, either Simpson or trapezoidal rules to approximate the different integral terms appearing in the fully discretized problem can be used.

So, if $N_{\text {dof }}$ denotes the dimension of the finite elements space, in the case without early retirement we have to solve the linear system with $N_{\text {dof }}$ unknowns

$$
M_{h} V_{h}^{n}=b_{h}^{n-1},
$$

at each time step, where matrix $M_{h}$ does not depend on time. Notice that the dependence on time of the PDE operator is restricted to the velocity and this term has been discretized by characteristics method, which combined with the previously described Crank-Nicolson scheme leads to a time-independent matrix.

4.2. Augmmented Lagrangian active set (ALAS) algorithm. The Augmented Lagrangian Active Set (ALAS) algorithm proposed in [14] is here applied to the fully discretized in time and space mixed formulation (4.2)-(4.3). More precisely, after this discretization the discrete problem can be written in the form:

$$
M_{h} V_{h}^{n}+P_{h}^{n}=b_{h}^{n-1}
$$

with the discrete complementarity conditions

$$
V_{h}^{n} \geq \bar{\Psi}_{h}^{n}, \quad P_{h}^{n} \leq 0, \quad\left(V_{h}^{n}-\bar{\Psi}_{h}^{n}\right) P_{h}^{n}=0,
$$

where $P_{h}^{n}$ denotes the vector of the multiplier values and $\bar{\Psi}_{h}^{n}$ denotes the vector of the nodal values defined by function $\bar{\Psi}$.

The basic iteration of the ALAS algorithm consists of two steps. In the first one the domain is decomposed into active and inactive parts (depending on whether the constraints are active or not), and in the second step, a reduced linear system associated to the inactive part is solved. Thus, we use the algorithm for unilateral problems, which are based on the augmented Lagrangian formulation.

First, for any decomposition $\mathcal{N}=\mathcal{I} \cup \mathcal{J}$, where $\mathcal{N}:=\left\{1,2, \ldots N_{\text {dof }}\right\}$, let us denote by $\left[M_{h}\right]_{\mathcal{I} \mathcal{I}}$ the principal minor of matrix $M_{h}$ and by $\left[M_{h}\right]_{\mathcal{I} \mathcal{J}}$ the co-diagonal block indexed by $\mathcal{I}$ and $\mathcal{J}$. Thus, for each time $t_{n}$, the ALAS algorithm computes not only $V_{h}^{n}$ and $P_{h}^{n}$ but also a decomposition $N=\mathcal{J}^{n} \cup \mathcal{I}^{n}$ such that

$$
\begin{aligned}
M_{h} V_{h}^{n}+P_{h}^{n} & =b_{h}^{n-1}, & & \\
{\left[P_{h}^{n}\right]_{j}+\beta\left[V_{h}^{n}-\bar{\Psi}\right]_{j} } & \leq 0, & & \forall j \in \mathcal{J}^{n}, \\
{\left[P_{h}^{n}\right]_{i} } & =0, & & \forall i \in \mathcal{I}^{n},
\end{aligned}
$$

for a given positive parameter $\beta$. In the above equations, $\mathcal{I}^{n}$ and $\mathcal{J}^{n}$ are the inactive and the active sets at time $t_{n}$ respectively. More precisely, the iterative algorithm builds sequences $\left\{V_{h, m}^{n}\right\}_{m},\left\{P_{h, m}^{n}\right\}_{m}$, $\left\{\mathcal{I}_{m}^{n}\right\}_{m}$ and $\left\{\mathcal{J}_{m}^{n}\right\}_{m}$, converging to $V_{h}^{n}, P_{h}^{n}, \mathcal{I}^{n}$ and $\mathcal{J}^{n}$, by means of the following steps: 
1. Initialize $V_{h, 0}^{n}=\bar{\Psi}_{h}^{n}$ and $P_{h, 0}^{n}=\min \left\{b_{h}^{n}-M_{h} V_{h, 0}^{n}, 0\right\} \leq 0$. Choose $\beta>0$. Set $m=0$.

2. Compute

$$
\begin{aligned}
Q_{h, m}^{n} & =\min \left\{0, P_{h, m}^{n}+\beta\left(V_{h, m}^{n}-\bar{\Psi}_{h, m}^{n}\right)\right\}, \\
\mathcal{J}_{m}^{n} & =\left\{j \in \mathcal{N},\left[Q_{h, m}^{n}\right]_{j}<0\right\} \\
\mathcal{I}_{m}^{n} & =\left\{i \in \mathcal{N},\left[Q_{h, m}^{n}\right]_{i}=0\right\} .
\end{aligned}
$$

3. If $m \geq 1$ and $J_{m}^{n}=J_{m-1}^{n}$ then convergence is achieved. Stop.

4. Let $V$ and $P$ be the solution of the linear system

$$
\begin{aligned}
& M_{h} V+P=b_{h}^{n-1}, \\
& P=0 \text { on } \mathcal{I}_{m}^{n} \text { and } V=\bar{\Psi}_{h, m}^{n} \text { on } \mathcal{J}_{m}^{n} .
\end{aligned}
$$

Set $V_{h, m+1}^{n}=V, P_{h, m+1}^{n}=\min \{0, P\}, m=m+1$ and go to Step 2 .

It is important to notice that, instead of solving the full linear system in (4.18), for $\mathcal{I}=\mathcal{I}_{m}^{n}$ and $\mathcal{J}=\mathcal{J}_{m}^{n}$ the following reduced one on the inactive set is solved:

$$
\begin{aligned}
{\left[M_{h}\right]_{\mathcal{I} \mathcal{I}}[V]_{\mathcal{I}} } & =\left[b^{n-1}\right]_{\mathcal{I}}-\left[M_{h}\right]_{\mathcal{I} \mathcal{J}}[\bar{\Psi}]_{\mathcal{J}}, \\
{[V]_{\mathcal{J}} } & =[\bar{\Psi}]_{\mathcal{J}} \\
P & =b^{n-1}-M_{h} V .
\end{aligned}
$$

In [14], it is proved the convergence of the algorithm in a finite number of steps for a Stieltjes matrix (i.e. a real symmetric positive definite matrix with negative off-diagonal entries, cf. [21]) and a suitable initialization (the same we consider in this paper). They also proved that $\mathcal{I}_{m} \subset \mathcal{I}_{m+1}$. Nevertheless, a Stieltjes matrix can be only obtained for linear elements but never for the here used quadratic elements because we have some positive off-diagonal entries coming from the stiffness matrix (actually we use a lumped mass matrix). However, we have obtained good results by using ALAS algorithm with quadratic finite elements.

5. Numerical examples. In this section we present some numerical results to illustrate the performance of the proposed numerical methods. For this purpose, we have considered the following model parameters:

$$
\begin{aligned}
& \sigma=0.1, \theta=0.025, r=0.025, a=0.75, T_{r}=40, T_{0}=15, \\
& n_{y}=30, k_{1}=0.5, \mu_{d}=0.025, \mu_{w}=0.2, \alpha_{d}=1, \alpha_{w}=0 .
\end{aligned}
$$

The bounded computational domain is defined by the values $x_{1}^{\infty}=40$ and $x_{2}^{\infty}=40$. Concerning the quadrangular finite element meshes data, in Table 5.1 the number of nodes and elements of the referred meshes in forthcoming tables and figures are shown. Moreover, we set $\beta=10000$ in the ALAS algorithm.

Also different numbers of time steps have been considered in different tests, thus for given values of $S$ and $I$, Table 5.2 illustrates the convergence of the proposed numerical methods as soon as the meshes is refined in time and space for $t=38$ and $(S, I)=(25,20)$. Notice that the point $(S, I)=(25,20)$ is located at the region where early retirement is not optimal. For the same point, in Tables 5.3 and 5.4 the results for $t=T_{r}-n_{y}=10$ and $t=0$ are shown, respectively.

Figure 5.1 displays the computed retirement benefits at $t=38$ for 10000 time steps and Mesh 96 from Table 5.1, the information about the numerical solution is completed with Figure 5.2 showing the 


\begin{tabular}{|l|r|r|}
\hline & N. Elem & N. Nodes \\
\hline Mesh 12 & 144 & 625 \\
\hline Mesh 24 & 576 & 2401 \\
\hline Mesh 48 & 2304 & 9409 \\
\hline Mesh 96 & 9216 & 37249 \\
\hline
\end{tabular}

Table 5.1: FEM meshes data

\begin{tabular}{|c|c|c|c|c|}
\hline time steps & Mesh 12 & Mesh 24 & Mesh 48 & Mesh 96 \\
\hline $\mathbf{1 2 5 0}$ & 1.703567 & 1.703345 & 1.703326 & 1.703321 \\
\hline $\mathbf{2 5 0 0}$ & 1.703773 & 1.703519 & 1.703491 & 1.703488 \\
\hline $\mathbf{5 0 0 0}$ & 1.703871 & 1.703615 & 1.703581 & 1.703577 \\
\hline $\mathbf{1 0 0 0 0}$ & 1.703923 & 1.703663 & 1.703626 & 1.703622 \\
\hline
\end{tabular}

Table 5.2: Retirement benefits at time $t=38$ at the mesh point $(S, I)=(25,20)$ when the parameters $\sigma=0.1, \theta=0.025, r=0.025, a=0.75, n_{y}=30, k_{1}=0.5, \mu_{d}=0.025, \mu_{c}=0.2, \alpha_{d}=1$ and $\alpha_{w}=0$ are considered

\begin{tabular}{|c|c|c|c|c|}
\hline time steps & Mesh 12 & Mesh 24 & Mesh 48 & Mesh 96 \\
\hline $\mathbf{1 2 5 0}$ & 1.703567 & 1.703345 & 1.703326 & 1.703321 \\
\hline $\mathbf{2 5 0 0}$ & 1.703773 & 1.703519 & 1.703491 & 1.703488 \\
\hline $\mathbf{5 0 0 0}$ & 1.703871 & 1.703615 & 1.703581 & 1.703577 \\
\hline $\mathbf{1 0 0 0 0}$ & 1.703923 & 1.703663 & 1.703626 & 1.703622 \\
\hline
\end{tabular}

Table 5.3: Retirement benefits at time $t=10$ and the mesh point $(S, I)=(25,20)$ when the parameters $\sigma=0.1, \theta=0.025, r=0.025, a=0.75, n_{y}=30, k_{1}=0.5, \mu_{d}=0.025, \mu_{c}=0.2, \alpha_{d}=1$ and $\alpha_{w}=0$ are considered

\begin{tabular}{|c|c|c|c|c|}
\hline time steps & Mesh 12 & Mesh 24 & Mesh 48 & Mesh 96 \\
\hline $\mathbf{1 2 5 0}$ & 2.800543 & 2.800665 & 2.800674 & 2.800683 \\
\hline $\mathbf{2 5 0 0}$ & 2.800349 & 2.800472 & 2.800479 & 2.800481 \\
\hline $\mathbf{5 0 0 0}$ & 2.800288 & 2.800366 & 2.800373 & 2.800374 \\
\hline $\mathbf{1 0 0 0 0}$ & 2.800295 & 2.800314 & 2.800319 & 2.800321 \\
\hline
\end{tabular}

Table 5.4: Retirement benefits at time $t=0$ and the mesh point $(S, I)=(25,20)$ when the parameters $\sigma=0.1, \theta=0.025, r=0.025, a=0.75, n_{y}=30, k_{1}=0.5, \mu_{d}=0.025, \mu_{c}=0.2, \alpha_{d}=1$ and $\alpha_{w}=0$ are considered

multiplier. Thus, the region where the multiplier is negative coincides with the region where is optimal to exercise the early retirement option. Moreover, Figure 5.3 shows the early retirement (coincidence) region in red and the non early retirement (non coincidence) region in blue, the curve separating both region being the optimal retirement boundary (free boundary). Figure 5.4 shows the computed retirement benefits at $t=T_{r}-n_{y}=10$ for 10000 time steps and Mesh 96, as expected the early retirement region is empty and we have obtained that the computed multiplier is equal to zero.

The evolution in time of the optimal retirement boundary is depicted in Figure 5.5. The critical salary 
for early retirement is represented as a function $\bar{S}=\bar{S}(t, I)$ of time $t$ and the cumulative salary $I$.

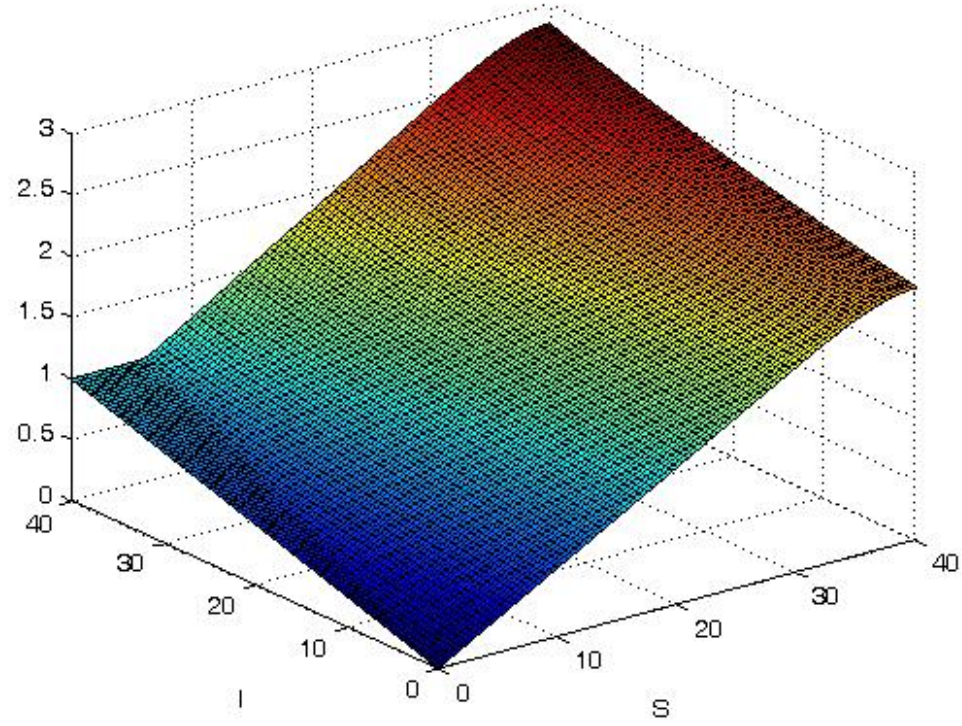

Fig. 5.1: Retirement benefits at time $t=38$ when the parameters $\sigma=0.1, \theta=0.025, r=0.025$, $a=0.75, n_{y}=30, k_{1}=0.5, \mu_{d}=0.025, \mu_{w}=0.2, \alpha_{d}=1$ and $\alpha_{w}=0$ are considered

Table 5.5 shows some numerical results obtained for the pension plan benefits $V$ and the multiplier $P$. More precisely, for different values of $(S, I)$, first row shows the value of $V$ when early retirement of the pension member is not allowed, while second and third rows indicate the value of $V$ and $P$ when early retirement is allowed. As expected, the value of $V$ is always greater in case of allowing early retirement. Also note that only the point $(S, I)=(4,10)$ belongs to the region where it is optimal not to retire as indicated by the null value of the multiplier.

\begin{tabular}{|c|c|c|c|c|}
\hline & $(S, I)=(1.2,15)$ & $(S, I)=(1.2,22.5)$ & $(S, I)=(2.4,30)$ & $(S, I)=(4,10)$ \\
\hline \hline No ER & 0.29442368 & 0.40814817 & 0.58884736 & 0.37288162 \\
\hline \hline ER & 0.36964285 & 0.55446428 & 0.73928571 & 0.37488181 \\
\hline Mult. & $-3.4539 \times 10^{-6}$ & $-6.9085 \times 10^{-6}$ & $-6.9078 \times 10^{-6}$ & 0 \\
\hline
\end{tabular}

Table 5.5: Retirement benefits at different points when time $t=38$ and the parameters $\sigma=0.1$, $\theta=0.025, r=0.025, a=0.75, n_{y}=30, k_{1}=0.5, \mu_{d}=0.025, \mu_{w}=0.2, \alpha_{d}=1$ and $\alpha_{w}=0$ are considered

\section{REFERENCES}

[1] A. Bermúdez, M. R. Nogueiras and C. VÁzquez, Numerical analysis of convection-diffusion-reaction pro-blems with higher order characteristics finite elements. Part I: Time discretization, SIAM J. Numer. Anal., 44 (2006), pp. 1829-1853. 


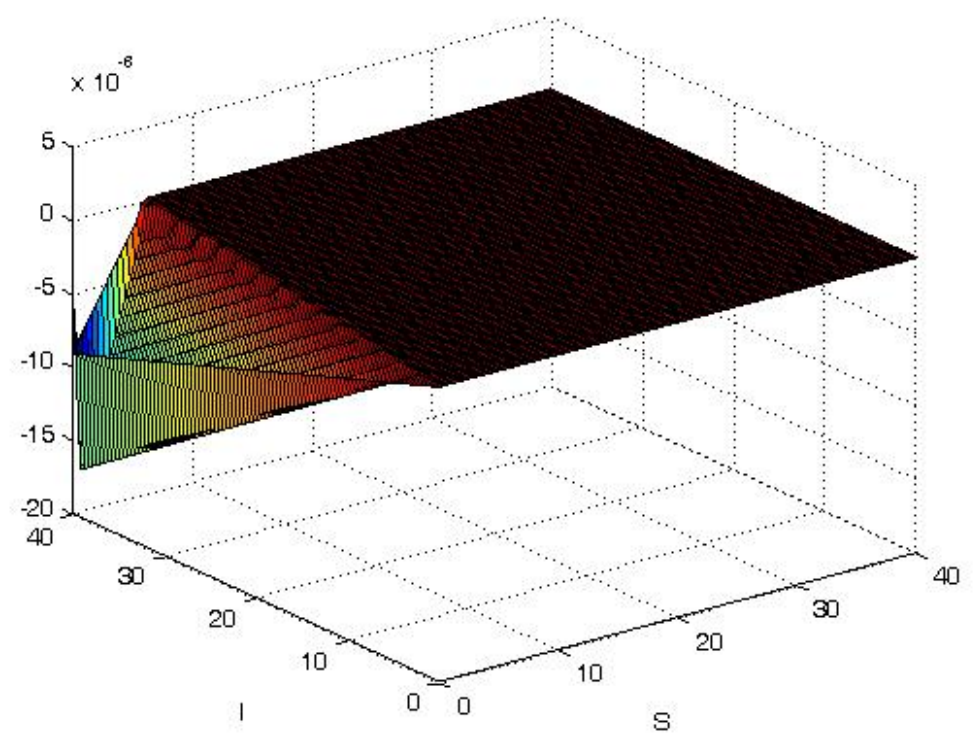

Fig. 5.2: Multiplier value at time $t=38$ when the parameters $\sigma=0.1, \theta=0.025, r=0.025, a=0.75$, $n_{y}=30, k_{1}=0.5, \mu_{d}=0.025, \mu_{w}=0.2, \alpha_{d}=1$ and $\alpha_{w}=0$ are considered

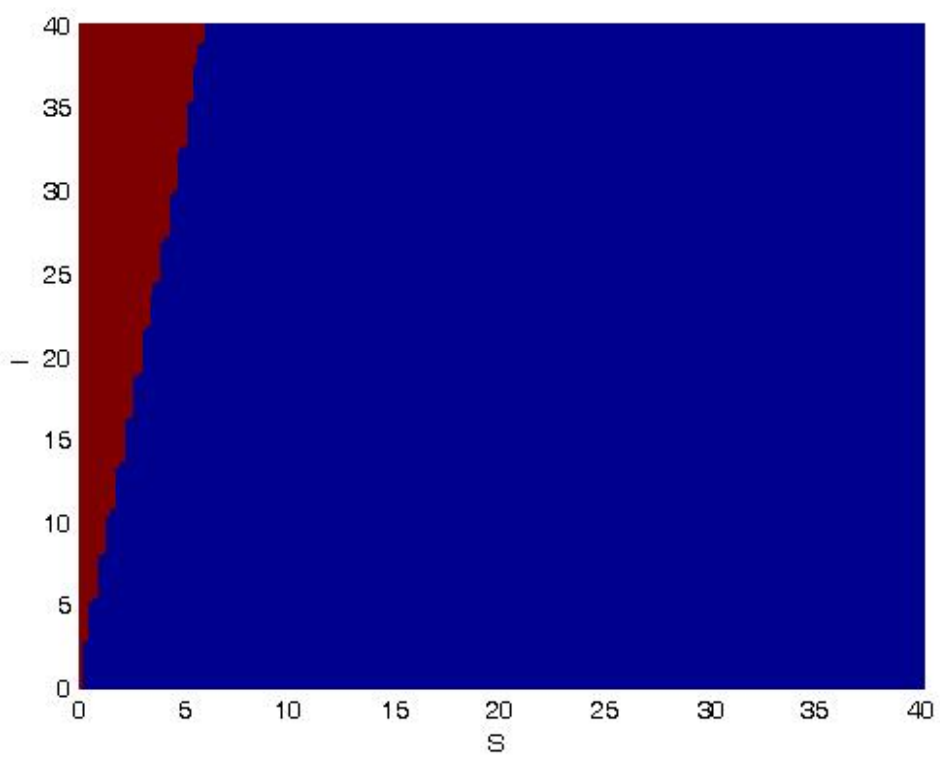

Fig. 5.3: Free boundary at time $t=38$ when the parameters $\sigma=0.1, \theta=0.025, r=0.025, a=0.75$, $n_{y}=30, k_{1}=0.5, \mu_{d}=0.025, \mu_{w}=0.2, \alpha_{d}=1$ and $\alpha_{w}=0$ are considered 


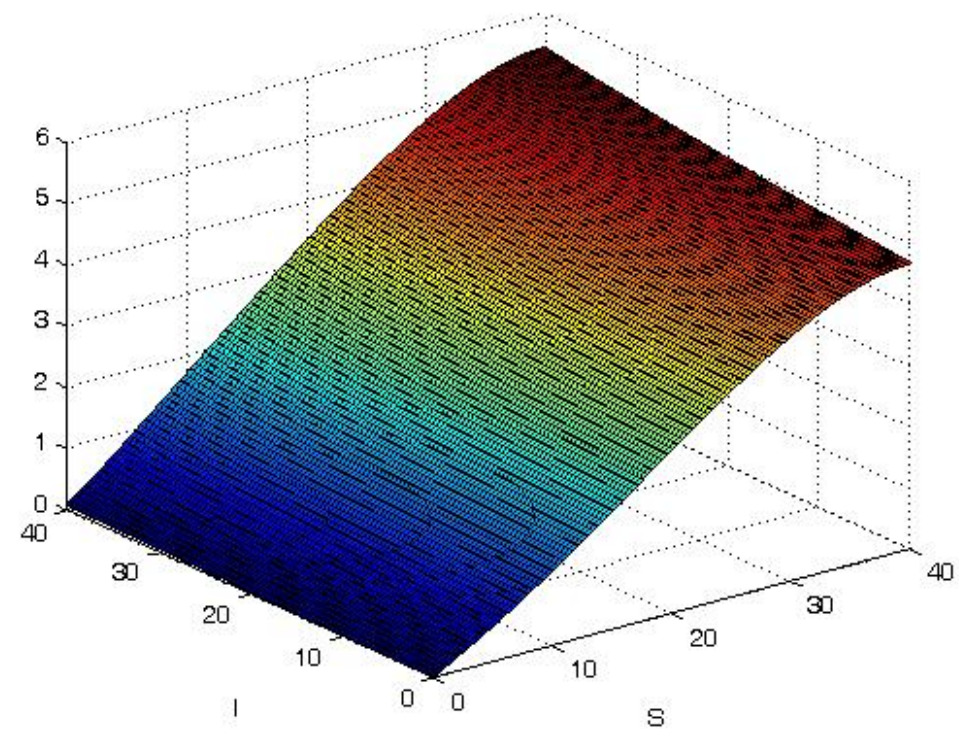

Fig. 5.4: Retirement benefits at time $t=10$ when the parameters $\sigma=0.1, \theta=0.025, r=0.025$, $a=0.75, n_{y}=30, k_{1}=0.5, \mu_{d}=0.025, \mu_{w}=0.2, \alpha_{d}=1$ and $\alpha_{w}=0$ are considered

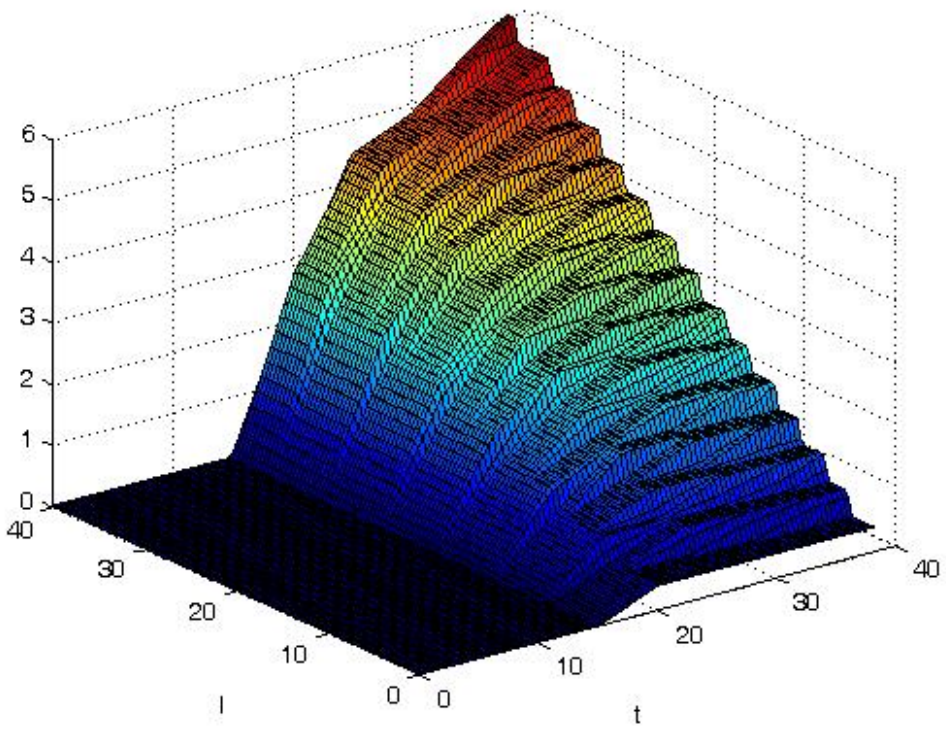

Fig. 5.5: Optimal retirement boundary $S=\bar{S}(t, \bar{I}(t))$ when the parameters $\sigma=0.1, \theta=0.025, r=0.025$, $a=0.75, n_{y}=30, k_{1}=0.5, \mu_{d}=0.025, \mu_{w}=0.2, \alpha_{d}=1$ and $\alpha_{w}=0$ are considered 
[2] A. Bermúdez, M. R. Nogueiras and C. VÁzquez, Numerical analysis of convection-diffusion-reaction problems with higher order characteristics finite elements. Part II: Fully discretized scheme and quadrature formulas, SIAM J. Numer. Anal., 44 (2006), pp. 1854-1876.

[3] A. Bermúdez, M. R. Nogueiras and C. VÁzquez, Numerical solution of variational inequalities for pricing Asian options by higher order Lagrange-Galerkin methods, Appl. Numer. Math., 56 (2006), pp. 1256-1270.

[4] A. Bermúdez, M. R. Nogueiras and C. VÁzquez, Comparison of two algorithms to solve a fixed-strike Amerasian options pricing problem, in International Series in Numerical Mathematics, 154, 2006, pp. 95-106.

[5] Z. Bodie, Pensions as retirement income insurance, J. of Economic Literature, 18 (1990), pp. 28-49.

[6] N. L. Jr. Bowers, H. V. Gerber, J. C. Nickman and C. J. Nesbitt, Actuarial Mathematics, Society of Actuaries, Schaumburg, Illinois, 1997.

[7] M.C. Calvo-Garrido and C. VÁzquez, Pricing pension plans based on average salary without early retirement: PDE modelling and numerical solution, to appear in J. of Computational Finance, (2012).

[8] E. Chevalier, Optimal early retirement near the expiration of a pension plan, Finance Stoch., 10 (2006), pp. 204221.

[9] M. Di Francesco And A. PAscucci, On a class of degenerate parabolic equations of Kolmogorov type, AMRX Appl. Math. Res. Express, 3 (2005), pp. 77-116.

[10] M. Di Francesco, A. Pascucci and S. Polidoro, The obstacle problem for a class of hypoelliptic ultraparabolic equations, Proc. R. Soc. London Ser. A: Math. Phys. Eng. Sci., 464 (2008), pp. 155-176.

[11] P. Faurre, Analyse numérique: Notes d'optimisation, École Polytechnique, Palaiseau, 1988.

[12] M. Frentz, K. Nyström, A. Pascucci, and S. Polidoro, Optimal regularity in the obstacle problem for Kolmogorov operators related to American Asian options, Math. Ann., 347 (2010), pp. 805-838.

[13] A. Friedman And W. Shen, A variational inequality approach to financial valuation of retirement benefits based on salary, Finance and Stoch., 6 (2002), pp. 273-302.

[14] T. KärkKäInen, K. Kunisch, And P. TARVAinen, Augmented Lagrangian active set methods for obstacle problems, J. Optim. Theory Appl., 119 (2003), pp. 499-533.

[15] E. Lanconelli And S. Polidoro, On a class of hypoelliptic evolution operators, Rend. Sem. Mat. Univ. Politec. Torino, 52 (1994), pp. 29-63.

[16] L. Monti, A. Pascucci, Obstacle problem for arithmetic Asian options, C.R. Acad. Sci. Paris, Ser. I, 347 (2009), pp. 1443-1446.

[17] A. PASCUCCI, Free boundary and optimal stopping problems for American Asian options, Finance Stoch., 12 (2008), pp. 21-41.

[18] O. Pironneau, On the transport-diffusion algorithm and its application to the Navier-Stokes equations, Numer. Math., 38 (1982), pp. 309-332.

[19] S. Polidoro, On a class of ultraparabolic operators of Kolmogorov-Fokker-Planck type, Matematiche (Catania), 49 (1994), pp. 53-105.

[20] M. SHERRIS AND W. SHEn, Financial valuation of retirement benefits based on salary, in Quantitative Methods in Finance Conference, Sidney, 1999.

[21] R. S VArga, Matrix iterative analysis, Prentice-Hall Inc., Englewood Cliffs, N.J., 1962. 